\title{
Astronautics \& Astronomy - a Profitable Partnership
}

\author{
Othon Cabo Winter ${ }^{1}$
}

$\mathrm{T}$ he connection between Astronautics and Astronomy is naturally straightforward. Astronomy is one of the oldest scientific areas of the human development, for thousands of years. On the other side, Astronautics is much younger (a few centuries), but it actually started in the dreams and in the poetic literature well before we have had technology to accomplish a remarkable fact as the launching of the first artificial satellite in orbit of the Earth.

Since then, more than half century ago, a huge number of artificial satellites have been launched. They are used for all kind of Earth monitoring, searching, communicating etc. They became a need for the current modern society. They became so important that in the last decades we started to worry about the space debris we produce and the threat they make on the indispensable new satellites to come.

Together with all that, we live on what has been called "The Space Age", the time of space exploration. Soon after the first artificial satellites we had probes sent to the Moon and to other nearby planets. Then, the fantastic race to the Moon has occurred, which provoked a significant technological advancement and also produced scientific knowledge. The rocks brought from the Moon induced a different thought about its origin. Information on their mineral and chemical composition produced models showing that our Moon could have been created from a giant impact between a Mars-size body (called Theia) and the proto-Earth.

Afterwards, the space exploration kept increasing. Initially there were spacecraft that made flybys on the planets (as the Voyager I and II, for example). Then, in the last decades, the long-duration explorations have started. A very good example is the Cassini/Huygens mission, which is exploring the Saturnian system (Saturn, its rings and moons) for about ten years. The spacecraft is very sophisticated, with a large number of state-of-the-art instruments. It also carried a probe that was dropped collecting data through the atmosphere of Titan (the largest satellite). These data is helping to build models in order to try to understand how the atmosphere of our planet was in the past. There has been so many incredible space missions to Jupiter, Mars, Mercury, the Sun, asteroids... Just along the last 12 months there were (and they are still going on) two amazing missions: Rosetta and New Horizons.

The comet 67P/Churyumov-Gerasimenko is being explored by the spacecraft Rosetta, which also carried a probe named Philae. The probe was placed on the surface of its nucleus to make measurements on its site, while the spacecraft is maintained nearby the comet (making other kinds of measurements). This comet is a small highly-irregular shaped body (far away from a spheroid body) with a largest axis of about six kilometers and a shortest axis of about two kilometers. Then, after a ten-year journey, being at a distance of about three times the Sun-Earth distance, they managed to place Philae, a hundred-kilogram probe, on the surface of this comet.

The New Horizons mission has completely different goals. The main one is the flyby of the Pluto-Charon system. The most distant celestial bodies ever visited by a man-made spacecraft. This system is located about 32 times the Sun-Earth distance. In order to reach such a long distance in just ten years, the spacecraft needed a very high velocity. Therefore, it was planned in a way to be the fastest spacecraft ever made. In the beginning of July 2015, it passed by Pluto at a distance of just about 12,000 kilometers, with a velocity of 23 kilometers per second, with a command signal delay of more than 4 hours (one way).

What all these space missions have in common? They were originated from a scientific perspective. Their main goal was astronomical, the exploration of the yet unknown, the search and discovery of information that was unavailable from Earth. On the other hand, they required projects (planning, development and execution) with creativity and novelties that promoted

1. Universidade Estadual Paulista "Júlio de Mesquita Filho" - Faculdade de Engenharia de Guaratinguetá - Grupo de Dinâmica Orbital \& Planetologia - Guaratinguetá/SP - Brazil. Author for correspondence: Othon Cabo Winter | Avenida Ariberto Pereira da Cunha, 333 - Portal das Colinas | CEP: $12.516-410$ - Guaratinguetá/SP - Brazil | Email: ocwinter@gmail.com 
strong advancements in Astronautics. Therefore, in all these cases, astronomical needs (scientific goals) imposed astronautical challenges, and both evolved significantly.

Apart from these major space missions, there are other fields where Astronautics and Astronomy cooperate and evolve together. In the field of Celestial Mechanics there is a productive two-way collaboration between Dynamical Astronomy and Astrodynamics. Along the last ten years Brazilian researchers from the two backgrounds have been collaborating in a way to bring knowledge from one side to improve the other and vice versa.

For instance, the problem of gravitational capture, or ballistic capture, has been extensively used in order to make the insertion of a spacecraft around a planet or a natural satellite (Belbruno and Miller 1990, 1993; Vieira Neto and Prado 1998; Vieira Neto 1998; Yamakawa 1992). This mechanism is also frequently considered in the study on the origin of giant planets' irregular natural satellites (Vieira Neto and Winter 2001; Agnor and Hamilton 2006). Irregular natural satellites are those whose orbits are highly eccentric, inclined and far from the planet (Peale 1999). The approaches adopted in Astronautics have been used in Astronomy and vice versa.

Another interesting example is related to the study of stability around the triple asteroid system 2001SN263. Since this is the target of a proposed Brazilian space mission (Sukhanov et al. 2010; Macau et al.2010), studies were made in order to identify the stable regions. In the first study (Araújo et al. 2012), prograde trajectories were considered, whose main purpose was to know the regions where leftovers (debris) from the origin of the triple system may still reside. The second study (Araújo et al. 2015) considered retrograde trajectories. The interception of the two regions is a special region, which is stable for retrograde orbits but unstable for prograde ones. This special region is the most indicated for placing a spacecraft to explore the triple system, since it is stable to maintain the orbit of the spacecraft and is free of debris that could collide with the spacecraft. In these studies, the two goals (astronomical and astronautical) are interconnected. The resulting knowledge is useful for the control and guidance of the mission, and also for the understanding of the formation of a triple system like this one.

\section{REFERENCES}

Agnor CB, Hamilton DP (2006) Neptune's capture of its moon Triton in a binary-planet gravitational encounter. Nature 441(7090):192194. doi: 10.1038/nature04792

Araújo RAN, Winter OC, Prado AFBA, Sukhanov A (2012) Stability regions around the components of the triple system 2001 SN263. Mon Not R Astron Soc 423[4]:3058-3073. doi: 10.1111/j.13652966.2012.21101.x

Araújo RAN, Winter OC Prado AFBA (2015) Stable retrograde orbits around the triple system 2001 SN263. Mon Not R Astron Soc 449(4):4404-4414. doi: 10.1093/mnras/stv592

Belbruno EA, Miller JK (1990) A ballistic lunar capture trajectory for Japanese spacecraft Hiten. Jet Propulsion Lab., JPL IOM 312/90.41731, Internal Document, Pasadena, CA, USA.

Belbruno EA, Miller JK (1993) Sun-perturbed Earth-to-Moon transfers with ballistic capture. J Guid Control Dynam 16(4):770-775. doi: $10.2514 / 3.21079$

Macau EEN, Winter OC, Campos Velho HF, Sukhanov AA, Brum AGV, Ferreira JL, Hetem A, Sandonato GM, Sfair R (2010) The ASTER Mission: Exploring for the first time a triple system asteroid. Proceedings of the 62nd International Astronautical Congress; Cape Town, USA.

Peale S (1999) Origin and evolution of natural satellites. Annu Rev Astron Astrophys 37:533-602. doi: 10.1146/annurev. astro.37.1.533

Sukhanov AA, Campos Velho HF, Macau EEN, Winter OC (2010) The Aster project: flight to a near-Earth asteroid. Cosmic Res 48(5):443450. doi: 10.1134/S0010952510050114

Vieira Neto E (1998) Estudo numérico da captura gravitacional temporária utilizando o problema restrito de três corpos. PhD thesis. São José dos Campos: Instituto Nacional de Pesquisas Espaciais.

Vieira Neto E, Prado AFBA (1998) Time-of-flight analyses for the gravitational capture maneuver. J Guid Control Dynam 21(1):122126. doi: 10.2514/2.4207

Vieira Neto E, Winter OC [2001] Time analysis for temporary gravitational capture: satellites of Uranus. Astron J 122(1):440-448. doi: $10.1086 / 321101$

Yamakawa $\mathrm{H}$ (1992) On Earth-Moon transfer trajectory with gravitational capture. PhD thesis. Tokyo: University of Tokyo. 